\title{
Introduction to the Critical and Ethical Studies of Digital and Social Media Minitrack
}

\author{
Jennifer Pierre \\ University of California, Los Angeles \\ ip639@g.ucla.edu
}

\author{
Tonia Sutherland \\ University of Hawai'i at Mānoa \\ tsuther@,hawaii.edu
}

\begin{abstract}
This minitrack focuses on two themes: a) studies that critically interrogate how and when digital and social media (DSM) support existing power structures or realign power structures affecting underrepresented or marginalized groups, and b) studies that raise awareness of or illustrate the ethical issues associated with doing research on digital and social media. The papers in this minitrack discuss a wide range of topics related to these themes and span a variety of methodological and theoretical approaches, ranging from youth social media use and social media data analysis, to the intersection of fitness trackers and gender, to theories to consider in ethical studies of artificial intelligence research. The following introduction will review the overarching themes connecting these papers in additional detail, before discussing each paper's fit within these themes and finally providing individual descriptions of each paper.
\end{abstract}

\section{Key Themes in Critical and Ethical Studies of Digital and Social Media Research}

The area of critical and ethical studies of digital and social media research spans many sub-fields, including critical technology studies and critical information studies/information science, computer-mediated communication, human-computer interaction, critical, social, and community informatics, and more. The papers presented in this minitrack reflect these subfields through a variety of top-level disciplinary approaches, including through information studies and information science, communication, sociology and anthropology, and science and technology. A key challenge in bringing such varied disciplines together is to use these different lenses to focus on the same specific processes, specifically processes at the intersection of technology and society and the existing power structures, ethical concerns, and other infrastructural components that affect these sociotechnical contexts.

This minitrack is concerned less with the specific technical functioning of various digital and social media platforms, but rather the way in which these platforms exist within different societal contexts, and the societal and cultural factors that shape their use. With this area of focus comes the call to pose specific inquiries and best practices for engaging in this type of research and engaging with these platforms more broadly, which is reflected in the second overarching theme. As a result of the specific focus of this research area, the outputs of research papers range from intended policy implications to design considerations in addition to traditional scholarly contributions.

The papers represented in this minitrack each respond to the two major themes in different and focused ways. The first paper, "Better, Nicer, Clearer, Fairer: A Critical Assessment of the Movement for Ethical Artificial Intelligence and Machine Learning," addresses the second theme most strongly, opening up an important discussion on the ethical considerations surrounding artificial intelligence (AI) research. The authors view digital and social media as significant tools for communicating ideas about AI research that affect public perception and acceptance of certain company- based research behaviors. The second paper, "Inscribing Gender: A Duoethnographic Examination of Gendered Values and Practices in Fitness Tracker Design" continues on the thread of critical evaluation. However, it uses a unique methodological empirical approach to examine and demonstrate the effect of stereotypical and societal gender assumptions on the design and use of fitness tracking technology.

The third paper, "Evaluating Disagreement Between Domain Experts and Graduate Student Annotators," also falls under the realm of empirical research, using statistical and qualitative methods to reveal cultural influence on social media annotation 
and identifying the need for domain specific expertise in this area. Finally, the fourth paper in this minitrack, "Sex, Drugs, and Alcohol in the Digital Neighborhood: A multi-method analysis of online discourse amongst Black and Hispanic Youth," provides a detailed qualitative analysis of minority youth use of social media, specifically assessing the use of different platforms for discussing various health related topics and contributing to a larger discussion of the potential of social media in communicating about youth health concerns. Each of these papers contributes to a larger discussion on the power dynamics and infrastructural elements of social and digital media use, and represents an ongoing scholarly discussion and development of critical, thoughtful, and interdisciplinary work on socio-technical issues facing our society. The following sections will provide more detailed explanations of each paper.

\section{Better, Nicer, Clearer, Fairer: A Critical Assessment of the Movement for Ethical Artificial Intelligence and Machine Learning}

This paper uses frame analysis to examine recent highprofile statements endorsing principals of ethical design for artificial intelligence and machine learning. Guided by insights from values in design and the sociology of business ethics, Daniel M. Greene, Anna Lauren Hoffman, and Luke Stark uncover how these values statements set the grounding assumptions and terms of debate that make some conversations about ethical design possible while forestalling alternative visions.

\section{Inscribing Gender: A Duoethnographic Examination of Gendered Values and Practices in Fitness Tracker Design}

Using fitness trackers to generate and collect quantifiable data is a widespread practice aimed at better understanding one's health and body. The intentional design of fitness trackers as genderless or universal is predicated on masculinist design values and assumptions and does not result in "neutral" artifacts. Instead, ignoring gender in the design of fitness tracking devices marks a dangerous ongoing inattention to the needs, desires, lives, and life chances of women, as well as transgender and gender nonconforming persons. Authors Marika Cifor and Patricia Garcia utilize duoethnography, a methodology emphasizing personal narrative and dialogue, as a tool that promotes feminist reflexivity in the design and study of fitness tracking technologies. Using the Jawbone UP3 as an object of study, the authors present findings that illustrate the gendered physical and interface design features and discuss how these features reproduce narrow understandings of gender, health, and lived experiences.

\section{Evaluating Disagreement Between Domain Experts and Graduate Student Annotators}

Researchers in computer science have spent considerable time developing methods to increase the accuracy and richness of annotations. However, there is a dearth in research that examines the positionality of the annotator, how they are trained and what we can learn from disagreements between different groups of annotators. In this study, the authors use qualitative analysis, statistical methods, and computational methods to compare annotations between Chicagobased domain experts and graduate students who annotated a total of 1851 tweets with images that are apart of a larger corpora associated with the Chicago Gang Intervention Study, aimed at developing a computational system that detects aggression and loss among gang-involved youth in Chicago. The authors, Desmond Upton Patton, Philipp Blandfort, William Frey, Michael B. Gaskell, and Svebor Karaman, find evidence to support the study of disagreement between annotators and underscore the need for domain expertise when reviewing social media data from vulnerable populations. Implications for annotation and content moderation are also discussed in this piece.

\section{Sex, Drugs, and Alcohol in the Digital Neighborhood: A multi-method analysis of online discourse amongst Black and Hispanic Youth}

In the digital neighborhood, Black and Hispanic youth communicate their perspectives about a myriad of issues facing youth, including sex and substance use. This population of young people are also disproportionately burdened by negative outcomes associated with sex and substance use behaviors, even when their behavior is less risky. Given the increased likelihood of negative outcomes, Robin Stevens, Stephen Bonett, Kahaari Kenyatta, Deepti Chittamuru, and Amy Bleakley investigate how youth talk about these behaviors in their online social networks. This mixed methods study integrates a behavioral survey with a machine learning-supported, qualitative content analysis of one year of Facebook and Twitter posts 
from 50 participants, with feedback from a youth advisory board. Findings suggest that participants who post about alcohol. Women posted more about STIs, HIV and pregnancy. Posts around sexual behavior often discussed trust in sexual partnerships and gendered views of sex. Alcohol and marijuana were used to relieve stress, to handle personal grief and drank mere more likely to

community violence, and as a coping mechanism for general distress. Understanding how youth think about sexual risk and substance use can inform the design of more effective prevention efforts. 